\title{
The butterfly fauna of Wisconsin bogs: lessons for conservation
}

\author{
Ann B. Swengel $\cdot$ Scott R. Swengel
}

Received: 4 March 2010/Accepted: 2 September 2010/Published online: 18 September 2010

(C) The Author(s) 2010. This article is published with open access at Springerlink.com

\begin{abstract}
During 2002-2009, we surveyed butterflies at 73 bogs, 20 adjacent lowland roadsides, and 5 nearby upland roadsides in northern Wisconsin and three bogs in central Wisconsin, with additional observations from 1986 to 2001. Especially in northern Wisconsin, bogs are relatively unaffected by humans, but naturally comprise $<1 \%$ of the landscape. Bog specialist species composition varied by bog type (muskeg, kettlehole, coastal peatland). Specialist abundance also varied significantly both among bog types and within type among sites. A number of bog specialists frequently occurred in numerous examples of bogs, including all three types. But virtually no specialist individuals occurred in nearby upland roadsides. Northern Wisconsin bogs had similar specialist species richness compared to large barrens and heaths in the same region. Specialist species comprised a small proportion (10\%) of all butterfly species recorded in bogs, similar to proportions reported for specialists in tallgrass prairie and barrens. However, specialists accounted for nearly half the total individuals recorded in bogs, comparable to proportions of specialists found in less fragmented vegetation (barrens) and larger patches of favorably managed prairie, but far exceeding proportions observed in other highly fragmented prairie patches. A fundamental lesson may be that aiming to conserve typical ecosystems, even if native, and their average processes, leads to average (generalist) butterflies. Bogs have different vegetation types superimposed on each other, including bog, heath, forest, sedge meadow, and wet meadow associates in the same spots. Conservation management needs to avoid simplifying the vegetation to one layer, reducing specialist fauna. Long-term vegetative consistency, as in bogs, is advised for conservation management of specialist butterflies in other fragmented vegetations.
\end{abstract}

Keywords Bog - Specialist butterfly - Habitat fragmentation - Vegetative consistency · Ecosystem dynamism - Vegetative classification · Resource-based habitat definition . Habitat management

A. B. Swengel $(\bowtie) \cdot$ S. R. Swengel 909 Birch St, Baraboo, WI 53913, USA e-mail: swengel@naba.org 


\section{Introduction}

In temperate areas of North America and Europe, bog (peatland) vegetation is also rare, being naturally isolated and forming a low proportion of the natural landscape. Although often viewed as a long-lived successional stage between open water and forest in glaciated landscapes, peatlands can get reset to an earlier successional stage (Curtis 1959). Since bogs are well known for their relatively stable vegetations and insect faunas over the long term, they can also be viewed as a climax community (Spitzer et al. 1999; Spitzer and Danks 2006; Whitehouse 2006; Whitehouse et al. 2008). While often considered relatively uniform floristically both within and among sites, bogs actually contain many microhabitats (Väisänen 1992; Spitzer and Danks 2006; Turlure et al. 2009). In Wisconsin, bogs occur primarily in central and northern areas (Curtis 1959). Prior to European settlement, peatlands occurred in $<1 \%$ of the Wisconsin landscape (even counting only the northern third of the state), and most of that vegetation is still extant, with only $9 \%$ loss (Hoffman 2002), more lost in central than northern Wisconsin. Much of what is left, especially in northern Wisconsin, is relatively undegraded. Primary human impacts are roads and ditches; adjacent lands are more affected by timber harvesting, agriculture, and urbanization (pers. obs.). Conversion to cranberry agriculture and peat harvesting has occurred more in central Wisconsin bogs (Curtis 1959). By contrast, in Europe bog vegetation is much destroyed and degraded by human activities, along with the associated butterfly species of high conservation concern (Vandewoestijne and Baguette 2004; Schtickzelle et al. 2006; Spencer and Collins 2008; Turlure et al. 2009). The four bog-related vegetation types ranked highest in proportion of threatened butterfly species of their typical faunas (van Swaay et al. 2006).

In addition to observations by a few other lepidopterists, Nekola (1998) conducted a systematic survey of northern Wisconsin peatlands and their associated butterflies in 1996. He described three peatland types: muskeg (black spruce Picea mariana-cottongrass Eriophorum spissum-Carex oligosperma-Sphagnum savanna similar in elevation to surrounding uplands), kettlehole (Sphagnum-leatherleaf Chamaedaphne calyculata mats, often floating on lakes or sunk in depressions much lower than the surrounding landscape), and coastal peatland (tamarack Larix laricina-sedge, especially Carex lasiocarpa, mats with ridges of muskeg-like vegetation in estuaries along the Lake Superior coast). Many aspects of the flora are similar among these three types (Nekola and Kraft 2002), echoing Curtis's (1959) description of remarkably uniform bog structure and composition throughout the circumboreal region. Nekola (1998) nevertheless found significant differences in bog-obligate butterfly occurrence among these three bog types, and noted variation in flora amongst sites, especially kettleholes.

We have recorded butterflies in Wisconsin bogs since 1986. In this paper, we analyze these results to expand and extend Nekola's study in order to describe the fauna in relatively undegraded examples of a vegetation type occurring in naturally fragmented patches comprising relatively little of the landscape as a whole. During the same period, we conducted surveys of butterflies in prairies in seven midwestern states (Swengel 1996; Swengel and Swengel 1999a, 1999b, 2007) and Wisconsin pine barrens (Swengel 1998b; Swengel and Swengel 2005, 2007). Based on this field work and others' studies, we contrast the occurrence of specialist butterflies between vegetations altered and fragmented by humans (prairie, barrens: Curtis 1959; Samson and Knopf 1994; Riegler 1995) and naturally fragmented ones (bogs). These results should be useful for application to conservation of bog butterflies where they are vulnerable, and vulnerable butterflies in other fragmented vegetations. 


\section{Methods}

\section{Study regions}

The primary study region contains 73 bog sites scattered across an area $367 \mathrm{~km}$ east-west by $169 \mathrm{~km}$ north-south $\left(45.33-46.86^{\circ} \mathrm{N}, 88.21-92.56^{\circ} \mathrm{W}\right)$ in 12 contiguous counties spanning the entire breadth of northern Wisconsin. At 20 of these sites, we also surveyed the lowland (wetland) roadside ditch through or adjacent to the bog, and at five sites, we surveyed a more upland roadside corridor 20-350 $\mathrm{m}$ from the bog. In three large muskeg complexes, we counted surveys in each separate area as a separate site. In central Wisconsin, the three bogs in two contiguous counties (Jackson, Wood) are in an area $29 \mathrm{~km}$ east-west by $4 \mathrm{~km}$ north-south $\left(44.31-44.34^{\circ} \mathrm{N}, 90.19-90.56^{\circ} \mathrm{W}\right)$, which is $169 \mathrm{~km}$ south of the nearest study site in the northern study region. Nekola's (1998) study region comprises sites in and adjacent to the Lake Superior drainage basin in four contiguous counties (Ashland, Bayfield, Douglas, Iron) bordering the south lakeshore. This area is the north part of the west half of our northern study region. All our sites in those counties fall within his study region.

Within each study region, we biased toward high-quality examples of bog vegetation that were open to public visitation and efficient to gain access to and travel between due to clustering of sites and/or efficient routing among sites, including proximity to other kinds of sites of interest for other species. Nonetheless, peatlands often present difficulties of access both to them and across them, which reduces efficiency and amount of transect distance surveyed in a day. Roadside survey areas were selected because we noticed bog butterflies using them, they were en route to or from a bog survey route, or they appeared potentially of interest for either bog or other butterfly species.

\section{Surveys}

On 114 informal visits during 1986-2001 in both study regions (widely in the northern one), we recorded number of individuals by species per site, but did not standardize a route or record weather and effort (time and distance spent surveying). We began formal transect surveys in bogs in 1990, with most conducted during 2002-2009 (Table 1). In those last 8 years, we surveyed in a rotation through the western, central, and eastern sections of the

Table $1 \mathrm{~N}$ unit surveys and survey effort $(\mathrm{km}, \mathrm{h})$ in central and northern Wisconsin at 76 bog sites, 20 lowland roadsides, and 5 upland roadsides, from $23 \mathrm{Apr}$ to 12 Sep

\begin{tabular}{lrlrr}
\hline & $\begin{array}{l}\text { N unit } \\
\text { surveys }\end{array}$ & Years & $\mathrm{km}$ & $\mathrm{h}$ \\
\hline 1987-2001 & & & & \\
All sites & 50 & $1987-2001$ & 44.0 & 25.8 \\
Bog & 27 & $1990-2001$ & 21.5 & 13.1 \\
Lowland & 5 & $1999-2001$ & 3.1 & 2.1 \\
Upland & 18 & $1987-1996$ & 19.5 & 10.7 \\
& & $1998-2001$ & & \\
2002-2009 & & & & 377.2 \\
All sites & 1973 & $2002-2009$ & 921.9 & 321.3 \\
Bog & 1699 & $2002-2009$ & 806.5 & 42.5 \\
Lowland & 223 & $2002-2009$ & 80.5 & 13.5 \\
Upland & 51 & $2002-2009$ & 34.9 & \\
\hline
\end{tabular}


northern study region, trying to cover one section per weekend, or more if a section was missed the previous weekend and/or if time allowed. But we missed an occasional weekend per year due to weather or another commitment. Surveys occurred between 23 April and 12 September, usually early/mid May through early/mid August in most years. We also continued to record bog specialists informally observed in uplands and roadsides as we accessed bogs for formal surveys.

Our peatland transect surveys were like those in prairie and barrens, (similar to Pollard 1977 and as described in Swengel 1996, 1998b, and Swengel and Swengel 1997). We walked along a similar route per visit to a prairie, barrens, or bog at a slow pace (about $2 \mathrm{~km} / \mathrm{h}$ ) on parallel routes 5-10 m apart. We counted all adult butterflies observed ahead and to the sides, to the limit at which an individual could be identified, possibly with the aid of binoculars after detection, and tracked. A new sampling unit was designated whenever the vegetation along the route varied by management (type and/or years since last treatment), type (wet, mesic, dry), quality based on type of brush and diversity and abundance of native and exotic flora (undegraded, semi-degraded, highly degraded), and/or estimated macrosite canopy (grassland or open bog $<10 \%$, open savanna 10-24\%, closed savanna $25-49 \%$, forest opening 50-75\%). Routes crossed rather than followed ecotones and management boundaries to reduce edge effects, and were designed to minimize number of unit changes while covering representative areas of the site. Temperature, wind speed, percent cloud cover, percent time sun was shining, route distance, and time spent surveying were recorded for each unit. Data from each unit were kept separate. Surveys occurred during a wide range of times of day and weather, occasionally in intermittent light drizzle so long as butterfly activity was apparent, but not in continuous rain. All butterfly species found were counted, but survey times and locations were selected to study butterflies specialized to that vegetation.

In prairie and barrens, we categorized the species by habitat niche breadth (Swengel 1996, 1998b): (1) specialist (restricted or nearly so to herbaceous flora in prairie and/or savanna; sensitive to vegetative quality); (2) grassland species (widely inhabiting both native and degraded herbaceous flora); (3) generalist (inhabiting grassland and other vegetation types); and (4) immigrant (occurring in the study region during the growing season but unlikely to overwinter). In bogs, we used an analogous categorization applicable to this study region only, and these categories correspond approximately to those (in parentheses) described by Spitzer and Danks (2006) (Table 2): (1) bog specialist (tyrphobiontic) — restricted or nearly so to peatlands; (2) bog affiliate (tyrphophilic)—breeding in bogs as well as other vegetations (limited to species of north temperate or boreal affinity); (3) generalist (tyrphoneutral)_year-round resident primarily using vegetation other than bogs (if the species also breeds in bogs, its range includes non-montane areas well south of Wisconsin); and (4) immigrant (tyrphoxenous) — not a year-round resident of the region and unlikely to breed in bogs. In Wisconsin, the bog specialists are all at the southern end of their eastern North American range, with their known range not extending into the state immediately south of Wisconsin, but further east $L$. epixanthe and $L$. dorcas may occur in areas more southerly than Wisconsin (Opler 1992; Glassberg 1999; Nielsen 1999).

Analyses

As in Nekola and Kraft (2002), we identified the flight period per species per year by the first and last date we detected it across the region. Our population index is the peak survey count per site per brood, standardized to survey time, to create an observation rate (relative 
Table 2 Total individuals of all species in each species category in bogs, lowland roadsides, and upland roadsides during 2002-2009 on formal surveys, except of the 53 generalist, only the ten most frequently recorded and all confirmed non-native species (as in Layberry et al. 1998) are provided

\begin{tabular}{lllll}
\hline Species & Bog & Lowl & Upl & Notes \\
\hline
\end{tabular}

Bog specialists

L Bog copper

Lycaena epixanthe

$\mathrm{N}$ Bog fritillary

Boloria eunomia

N Jutta arctic

Oeneis jutta

$\mathrm{N}$ Freija fritillary

Boloria freija

N Frigga fritillary

Boloria frigga

N Purplish fritillary

Boloria montinus

L Dorcas copper

Lycaena dorcas

$\mathrm{N}$ Red-disked alpine

Erebia discoidalis

Bog affiliates

\begin{tabular}{|c|c|c|c|c|}
\hline L Callophrys augustinus & 4304 & 6 & 0 & Heaths, barrens, bogs \\
\hline N Coenonympha tullia & 587 & 162 & 3 & Wet/dry grassy areas \\
\hline Pi Colias interior & 301 & 223 & 9 & Heaths, barrens, forest openings \\
\hline N Satyrodes eurydice & 243 & 243 & 40 & Sedge meadows, marshes \\
\hline H Erynnis icelus & 168 & 7 & 2 & Open woodlands, barrens \\
\hline N Boloria selene & 123 & 89 & 9 & Wet meadows, marshes, bogs \\
\hline N Speyeria atlantis & 46 & 299 & 60 & Open woods \\
\hline N Chlosyne harrisii & 32 & 29 & 0 & $\begin{array}{l}\text { Wet meadows, bog edges, } \\
\text { marshes }\end{array}$ \\
\hline N Polygonia faunus & 1 & 0 & 0 & Forests \\
\hline \multicolumn{5}{|c|}{ Generalists (top ten species and all non-native species) } \\
\hline $\mathrm{H}^{\mathrm{a}}$ Thymelicus lineola & 1103 & 780 & 275 & Open grassy fields \\
\hline Pa Papilio canadensis & 389 & 31 & 13 & Deciduous woods \\
\hline H Poanes hobomok & 227 & 11 & 13 & Damp deciduous forest edges \\
\hline L Celastrina ladon & 176 & 15 & 11 & Brushy fields, forests and edges \\
\hline $\mathrm{N}$ Cercyonis pegala & 135 & 55 & 114 & Prairie, old field, meadow, bog \\
\hline N Phyciodes selenis & 126 & 245 & 61 & Moist open areas \\
\hline H Polites mystic & 85 & 156 & 60 & $\begin{array}{l}\text { Prairie swales, wet meadows, } \\
\text { marshes, streamsides }\end{array}$ \\
\hline Pi Colias philodice & 80 & 112 & 85 & $\begin{array}{l}\text { Clover fields, lawns, moist } \\
\text { meadows, fields }\end{array}$ \\
\hline H Erynnis juvenalis & 51 & 0 & 0 & Oak forest/scrub and adjacent \\
\hline $\mathrm{N}$ Limenitis arthemis & 44 & 53 & 6 & Deciduous/mixed woods \\
\hline $\mathrm{Pi}^{\mathrm{a}}$ Pieris rapae & 10 & 72 & 24 & Most kinds of open vegetations \\
\hline
\end{tabular}

$\begin{array}{llll}3671 & 530 & 2 & \text { 23 Jun-15 Aug } \\ 1084 & 0 & 1 & \text { 4 Jun-14 Jul } \\ 962 & 1 & 0 & \text { 12 May-10 Jul } \\ 722 & 0 & 0 & \text { 7 May-15 Jun } \\ 264 & 0 & 0 & \text { 19 May-20 Jun } \\ 188 & 1093 & 0 & \text { 25 Jul-23 Aug } \\ 158 & 379 & 0 & \text { 23 Jun-17 Aug } \\ 128 & 1 & 0 & \text { 10 May-14 Jun }\end{array}$

\section{s}


Table 2 continued

\begin{tabular}{lllll}
\hline Species & Bog & Lowl & Upl & Notes \\
\hline Immigrants & & & & \\
N Danaus plexippus & 342 & 21 & 50 & \\
N Vanessa virginiensis & 38 & 6 & 2 & \\
N Vanessa atalanta & 31 & 9 & 0 \\
N Junonia coenia & 30 & 1 & 0 \\
N Vanessa cardui & 10 & 2 & 0 \\
Pi Pontia protodice & 3 & 0 & 0 \\
N Euptoieta claudia & 2 & 0 &
\end{tabular}

Vegetative associations are from Opler and Krizek (1984)

Butterfly names follow Cassie et al. (2001)

Family abbreviations: $P a$ Papilionidae, $P i$ Pieridae, $L$ Lycaenidae, $N$ Nymphalidae, $H$ Hesperiidae

a Non-native

abundance) per hour per unit survey. Analysis was done with ABstat 7.20 software (1994 Anderson-Bell Corp, Parker, Colorado). Statistical significance was set at two-tailed $P<0.05$. Since significant results occurred at a frequency well above that expected due to spurious Type I statistical error, the critical $P$ value was not lowered further, as more Type II errors (biologically meaningful patterns lacking statistical significance) would be created than Type I errors eliminated. To test for significant skewing compared to distribution proportional to survey effort (h), we used the Chi Square Goodness of Fit test. Statistical tests in this study are non-parametric, which do not require any assumptions about how the data are distributed (e.g., normality).

\section{Results}

Specialists and affiliates comprised a relatively low proportion of total species in bogs-8 $(10.4 \%)$ and $9(11.7 \%)$, respectively, of 77 (Table 2) — but relatively high proportions and abundances of individuals (Table 3). They were also well represented in lowland roadsides, but much less so in nearby uplands (especially specialists, found only in upland roadsides $\leq 50 \mathrm{~m}$ from a bog (Table 3 ). Generalists steadily increased in relative abundance and proportion of individuals from bog to lowland roadside to upland roadside (Table 3). Immigrants were the lowest in relative abundance and proportion of individuals in all three site types, peaking in uplands (Table 3). B. montinus was the specialist most numerous in lowland roadsides (Table 2). Since it occurred in only one county (Douglas, the northwesternmost), we also provide results for lowland roadsides excluding this species (Table 3). In that case, the remaining specialist species were similarly abundant in bogs and lowland roadsides, but consistently decreased in proportion of total butterfly individuals from bog to lowland roadside to upland roadside. Total butterfly abundance was much lower in bogs, and similarly higher in lowland and upland roadsides (Table 3).

We recorded the same bog specialist and affiliate species in muskegs as reported in Nekola's (1998) study; additional species we recorded in kettleholes and coastal peatlands within Nekola's (1998) study region were infrequently encountered in only one or two sites per bog type (Table 4). 
Table 3 Mean, minimum, and maximum relative abundance (observation rate of individuals/h) of each species group and total individuals (including unidentified individuals), and proportion (\%) of each species group out of total individuals, per year during 2002-2009

\begin{tabular}{|c|c|c|c|c|c|c|c|c|c|}
\hline & \multicolumn{2}{|c|}{ Specialists } & \multicolumn{2}{|c|}{ Affiliates } & \multicolumn{2}{|c|}{ Generalists } & \multicolumn{2}{|c|}{ Immigrants } & \multirow{2}{*}{$\begin{array}{l}\text { Total } \\
\text { Rate }\end{array}$} \\
\hline & Rate & $\%$ & Rate & $\%$ & Rate & $\%$ & Rate & $\%$ & \\
\hline \multicolumn{10}{|l|}{ Bog } \\
\hline Mean & 21.6 & 44.90 & 18.2 & 34.00 & 9.3 & 18.20 & 1.4 & 2.90 & 54.3 \\
\hline Minimum & 15.9 & 24.90 & 5.2 & 15.30 & 4.9 & 10.70 & 0.1 & 0.20 & 32.4 \\
\hline Maximum & 29.9 & 68.00 & 30.8 & 52.80 & 22.8 & 35.80 & 5.5 & 9.30 & 74.5 \\
\hline \multicolumn{10}{|c|}{ Lowland roadsides } \\
\hline Mean & 51.3 & 33.00 & 24.9 & 17.90 & 68.2 & 48.30 & 1 & 0.70 & 149.3 \\
\hline Minimum & 20.2 & 15.50 & 14.3 & 7.70 & 35.6 & 34.40 & 0.2 & 0.10 & 106.4 \\
\hline Maximum & 140.7 & 56.40 & 47.8 & 27.60 & 97.6 & 63.10 & 2.9 & 2.30 & 255.9 \\
\hline \multicolumn{10}{|c|}{ Lowland roadsides (excluding Boloria montinus) } \\
\hline Mean & 22.7 & 18.50 & & & & & & & 120.7 \\
\hline Minimum & 3.8 & 4.30 & & & & & & & 78.6 \\
\hline Maximum & 63.1 & 36.70 & & & & & & & 178.1 \\
\hline \multicolumn{10}{|c|}{ Upland roadsides } \\
\hline Mean & 0.2 & 0.20 & 10 & 8.00 & 121.8 & 88.10 & 3.8 & 3.70 & 138.4 \\
\hline Minimum & 0 & 0.00 & 4.7 & 2.70 & 42.4 & 78.00 & 0 & 0.00 & 49 \\
\hline Maximum & 0.7 & 0.90 & 21.3 & 13.40 & 257 & 95.10 & 14.1 & 13.80 & 286.2 \\
\hline
\end{tabular}

Table 4 Presence of the ten peatland species analyzed by Nekola (1998) in the three bog types

\begin{tabular}{|c|c|c|c|c|c|c|}
\hline & \multicolumn{2}{|c|}{ Muskeg } & \multicolumn{2}{|c|}{ Kettlehole } & \multicolumn{2}{|c|}{ Coastal $^{\mathrm{a}}$} \\
\hline \multicolumn{7}{|l|}{ Bog specialists } \\
\hline L Lycaena epixanthe & $\mathrm{N}$ & $\mathrm{S} / \mathrm{S}$ & $\mathrm{N}$ & $\mathrm{S} / \mathrm{S}$ & $\mathrm{N}$ & S \\
\hline L Lycaena dorcas & $\mathrm{N}$ & $\mathrm{S} / \mathrm{S}$ & & & & $S$ \\
\hline $\mathrm{N}$ Boloria freija & $\mathrm{N}$ & $\mathrm{S} / \mathrm{S}$ & & $\mathrm{S} /$ & & $\mathrm{S}$ \\
\hline $\mathrm{N}$ Boloria frigga & $\mathrm{N}$ & $\mathrm{S} / \mathrm{S}$ & & & & \\
\hline N Boloria eunomia & $\mathrm{N}$ & $\mathrm{S} / \mathrm{S}$ & $\mathrm{N}$ & $\mathrm{S} / \mathrm{S}$ & $\mathrm{N}$ & $\mathrm{S}$ \\
\hline $\mathrm{N}$ Boloria montinus & $\mathrm{N}$ & $\mathrm{S} /$ & & & & \\
\hline N Erebia discoidalis & $\mathrm{N}$ & $\mathrm{S} / \mathrm{S}$ & & & & \\
\hline $\mathrm{N}$ Oeneis jutta & $\mathrm{N}$ & $\mathrm{S} / \mathrm{S}$ & $\mathrm{N}$ & $\mathrm{S} / \mathrm{S}$ & & $\mathrm{S}$ \\
\hline \multicolumn{7}{|l|}{ Bog affiliates } \\
\hline L Callophrys augustinus & $\mathrm{N}$ & $\mathrm{S} / \mathrm{S}$ & $\mathrm{N}$ & $\mathrm{S} / \mathrm{S}$ & $\mathrm{N}$ & $\mathrm{S}$ \\
\hline N Coenonympha tullia & $\mathrm{N}$ & $\mathrm{S} / \mathrm{S}$ & $\mathrm{N}$ & $\mathrm{S} / \mathrm{S}$ & $\mathrm{N}$ & $\mathrm{S}$ \\
\hline
\end{tabular}

By study reporting them: $N$ reported by Nekola and $S$ reported by this study in the northwest/northeast subregions; all Swengel additions are in kettleholes and coastal peatlands within Nekola's study region

Species occurrences in this study in bog types where they were not reported by Nekola (1998): Kettlehole 1 B. freija in East Wishbone Lake each in 3 years (all attempts in that unit). Coastal 1 B. freija in Bibon Lake in 1 year (not in another year); $1 O$. jutta in each of 5 years ( 0 in three other years) at Bibon Lake; $1 \mathrm{~L}$. dorcas at Bark Bay in 1993, 2 in 1997, 1 in 2001 (0 in 2005, 2007, 2008, and 2009) and 1 at Bibon Lake in each of 3 years ( 0 in two other years)

${ }^{\text {a }}$ Coastal peatlands occur only in the northwest 
Table 5 Bog specialist butterflies recorded during 2002-2009 in selected bog sites (not roadsides), grouped by bog type (subregion and counties in parentheses), maximum specialist species in range, and survey effort ( $\mathrm{km}$ and $\mathrm{h})$

\begin{tabular}{|c|c|c|c|c|}
\hline & $\begin{array}{l}\text { Total specialist } \\
\text { Species recorded } \\
\text { in range }^{\mathrm{a}}\end{array}$ & Maximum & $\begin{array}{l}\text { Survey } \\
\mathrm{km}\end{array}$ & $\begin{array}{l}\text { Survey } \\
\mathrm{h}\end{array}$ \\
\hline \multicolumn{5}{|c|}{ Muskegs (Northwest: Douglas) } \\
\hline Bear Lake & 8 & 8 & 44.5 & 18 \\
\hline Bear lake North & $7^{\mathrm{b}}$ & 8 & 33.9 & 13.1 \\
\hline Lyman Lake & 8 & 8 & 88.7 & 35.2 \\
\hline Milchesky Road & 8 & 8 & 40.8 & 16.1 \\
\hline Moose Junction & $7^{\mathrm{c}}$ & 8 & 29.2 & 11.5 \\
\hline \multicolumn{5}{|c|}{ Muskegs (Northwest: Ashland, Iron, Price) } \\
\hline Caroline Lake & $6^{\mathrm{b}}$ & 7 & 24.4 & 8.5 \\
\hline Forest Road 1372.3 & $6^{\mathrm{d}}$ & 7 & 18.2 & 7.9 \\
\hline Glidden & $6^{\mathrm{c}}$ & 7 & 55.8 & 21.5 \\
\hline \multicolumn{5}{|c|}{ Muskegs (Northeast: Forest) } \\
\hline Armstrong & 7 & 7 & 60.3 & 23.2 \\
\hline Forest Road $2182 \mathrm{~W}$ & 7 & 7 & 21.4 & 7.9 \\
\hline Forest Road 2414 & $5^{\mathrm{b}, \mathrm{e}}$ & 7 & 25.7 & 10.3 \\
\hline \multicolumn{5}{|c|}{ Kettleholes (Northwest: Bayfield interior) } \\
\hline East Crane Lake & 3 & 7 & 10.7 & 3.5 \\
\hline East Roger Lake & 3 & 7 & 14.8 & 6.3 \\
\hline East Wishbone & 4 & 7 & 23.9 & 9.9 \\
\hline Pine Lake & 2 & 7 & 8.1 & 2.9 \\
\hline \multicolumn{5}{|c|}{ Coastal Peatlands (Northwest: Bayfield coastal) } \\
\hline Bark Bay & 2 & 7 & 21.2 & 9.2 \\
\hline Bibon Lake & 5 & 7 & 15.9 & 6 \\
\hline Lost Creek & 2 & 7 & 16.9 & 7 \\
\hline Port Wing Boreal & 2 & 7 & 12.3 & 5.3 \\
\hline
\end{tabular}

${ }^{a}$ B. montinus was discovered in northwestern Wisconsin in the 1990s (Ferge 1992)

${ }^{\mathrm{b}}$ No B. frigga, ${ }^{\mathrm{c}}$ No E. discoidalis, ${ }^{\mathrm{d}}$ No L. dorcas, ${ }^{\mathrm{e}}$ No B. eunomia

In central Wisconsin, only three bog specialists were known to be in range (Glassberg 1999), indicating a depauperate fauna, although still remarkable for any species of boreal affinity to occur here. But in northern Wisconsin, bogs were not depauperate in specialists. In relatively little effort we recorded most or all possible bog specialist species in a number of muskegs (Table 5). This compared favorably with barrens specialists recorded in the same study region, at which we typically had similar or more survey effort (Table 6). Four specialists were widespread in bogs, occurring in most sites surveyed in 4 or more years (Table 7).

Specialists rarely occurred in nearby upland roadsides (Table 2) and all were found only in upland roadsides that were $\leq 50 \mathrm{~m}$ from a bog. Spring specialists rarely occurred in adjacent lowland roadsides, while the three summer species frequently occurred there (Table 2; Swengel and Swengel 2010), where they nectared at a variety of non-native flowers (Table 8) as well as native ones. By contrast, the seven immigrants were 
Table 6 Heath/barrens specialist butterflies recorded in sites (indicated by X) in northern study region, with statistics on survey characteristics

\begin{tabular}{|c|c|c|c|c|c|c|}
\hline County & $\begin{array}{l}\text { Dunbar } \\
\text { Barrens } \\
\text { Marinette }\end{array}$ & $\begin{array}{l}\text { Marinette } \\
\text { Co. Forest } \\
\text { Marinette }\end{array}$ & $\begin{array}{l}\text { Private } \\
\text { Forestry } \\
\text { Douglas }\end{array}$ & $\begin{array}{l}\text { Crex } \\
\text { Meadows } \\
\text { Burnett }\end{array}$ & $\begin{array}{l}\text { Burnett } \\
\text { Co. Forest } \\
\text { Burnett }\end{array}$ & $\begin{array}{l}\text { Moquah } \\
\text { Barrens } \\
\text { Bayfield }\end{array}$ \\
\hline Patch size (ha) & $535^{\mathrm{a}}$ & ca $18^{\mathrm{b}}$ & $>60^{\mathrm{b}}$ & $3240^{\mathrm{a}}$ & $30^{\mathrm{b}}$ & $2020^{\mathrm{a}}$ \\
\hline Survey effort (km) & 54.6 & 84.9 & 27 & 293.4 & 70.7 & 84.4 \\
\hline Survey effort (h) & 19.5 & 34.4 & 12.7 & 128.7 & 34.4 & 36.6 \\
\hline Earliest survey date & 15-May & 19-May & 8-May & 26-Apr & 26-Apr & 24-Apr \\
\hline Latest survey date & 14-Aug & 14-Aug & 12-Sep & 17-Aug & 17-Aug & 19-Sep \\
\hline Survey years & 2002-2009 & $\begin{array}{l}1998, \\
2002-2009\end{array}$ & 2003-2009 & $\begin{array}{l}1991- \\
2009\end{array}$ & $\begin{array}{l}1994- \\
2009\end{array}$ & $\begin{array}{l}1988- \\
2009\end{array}$ \\
\hline Pi Euchloe olympia & & & $\mathrm{X}$ & $\mathrm{X}$ & $\mathrm{X}$ & NA \\
\hline L Callophrys henrici & & & & & $\mathrm{X}$ & \\
\hline L Lycaeides idas & & $\mathrm{X}$ & NA & NA & NA & NA \\
\hline L L. melissa samuelis ${ }^{\mathrm{c}}$ & NA & NA & NA & $\mathrm{X}$ & $\mathrm{X}$ & NA \\
\hline N Chlosyne gorgone & & & $\mathrm{X}$ & $\mathrm{X}$ & $\mathrm{X}$ & NA \\
\hline N Oeneis chryxus & $\mathrm{X}$ & $\mathrm{X}$ & $\mathrm{X}$ & NA & NA & $\mathrm{X}$ \\
\hline $\mathrm{H}$ Erynnis martialis & NA & NA & $\mathrm{X}$ & $X$ & $\mathrm{X}$ & \\
\hline H Erynnis persius & NA & NA & NA & $\mathrm{X}$ & $\mathrm{X}$ & NA \\
\hline H Hesperia metea & $\mathrm{X}$ & & $X$ & & & \\
\hline H H. comma laurentina & $\mathrm{X}$ & $\mathrm{X}$ & & NA & NA & \\
\hline H Hesperia leonardus & $\mathrm{X}$ & $\mathrm{X}$ & $\mathrm{X}$ & $\mathrm{X}$ & $\mathrm{X}$ & $\mathrm{X}$ \\
\hline H Atrytonopsis hianna & NA & NA & $\mathrm{X}$ & $\mathrm{X}$ & $\mathrm{X}$ & \\
\hline Total observed & 4 & 4 & 7 & 7 & 8 & 2 \\
\hline Maximum in range & 8 & 8 & 9 & 9 & 9 & 7 \\
\hline
\end{tabular}

NA not applicable (not in known range per Opler and Krizek 1984)

${ }^{\text {a }}$ From Riegler (1995)

${ }^{\mathrm{b}}$ Estimate from personal observation and map

${ }^{c}$ Recognized as occurring in Wisconsin in the 1980s (Kuehn 1983); specimens had previously been attributed to L. idas

significantly over-represented in bogs in spring compared to summer, both as a group and by species for the five most frequently recorded ones (Table 9). Bogs were relatively nectar-rich in spring, more so than the roadsides, but nectar-poor in summer, when the roadsides were nectar-rich. We did not find non-native nectar in bogs but we did find the two non-native butterfly species in range there (Table 2).

During 2002-2009, number of individuals in each subgroup, and total individuals, deviated significantly from a distribution proportional to survey effort each year, indicating large fluctuations in abundance among years (Table 10, Chi Square Goodness of Fit $P=0.0000$ for each). Immigrants showed the most extreme variation: $53 \%$ of all immigrants found during this period occurred in 2007 (vs. 14\% expected), followed by $31 \%$ in 2006 (expected 13\%), compared to 1\% in 2008 (expected 13\%). Nonetheless, immigrants comprise a very small proportion of individuals and species observed in bogs (Table 2). 
Table $7 \mathrm{~N}$ sites where each bog specialist was detected, only counting bogs (not roadsides) surveyed during its flight period in northern Wisconsin during 2002-2009, for all bogs and by bog types ( $M$ muskeg, $K$ kettlehole, and $C$ coastal), and where undetected, tabulating all sites and only those surveyed four or more years

\begin{tabular}{|c|c|c|c|c|c|c|c|c|c|c|c|c|}
\hline & \multicolumn{4}{|c|}{ Detected } & \multicolumn{4}{|c|}{ Undetected (all) } & \multicolumn{4}{|c|}{ Undetected $(4+$ years $)$} \\
\hline & All & M & $\mathrm{K}$ & $\mathrm{C}$ & All & M & $\mathrm{K}$ & $\mathrm{C}$ & All & M & $\mathrm{K}$ & $\mathrm{C}$ \\
\hline Lycaena epixanthe & 40 & 27 & 9 & 4 & 5 & 5 & 0 & 0 & 1 & 1 & 0 & 0 \\
\hline Boloria eunomia & 32 & 21 & 7 & 4 & 11 & 7 & 4 & 0 & 1 & 1 & 0 & 0 \\
\hline Oeneis jutta & 30 & 27 & 2 & 1 & 5 & 0 & 2 & 3 & 4 & 0 & 1 & 3 \\
\hline Boloria freija & 26 & 24 & 1 & 1 & 18 & 14 & 2 & 2 & 2 & 2 & 0 & 0 \\
\hline Lycaena dorcas & 18 & 16 & 0 & 2 & 15 & 5 & 7 & 3 & 5 & 0 & 3 & 2 \\
\hline Boloria frigga ${ }^{\mathrm{a}}$ & 15 & 15 & 0 & 0 & 9 & 9 & 0 & 0 & 3 & 3 & 0 & 0 \\
\hline Erebia discoidalis & 15 & 15 & 0 & 0 & 22 & 15 & 3 & 4 & 5 & 5 & 0 & 0 \\
\hline Boloria montinus ${ }^{\mathrm{b}}$ & 6 & 6 & 0 & 0 & 19 & 15 & 3 & 1 & 0 & 0 & 0 & 0 \\
\hline
\end{tabular}

a Since only sites with dwarf birch (Betula pumila) had B. frigga detections, only sites with this plant were included for undetected sites

b All detections were in Douglas County and all non-detections were in other counties

Table 8 Nectar visits (defined as probing into flower) at non-native flowers in lowland roadsides (all also visited a variety of native flowers too)

\begin{tabular}{|c|c|c|c|}
\hline & $\begin{array}{l}\text { Lycaena } \\
\text { epixanthe }\end{array}$ & $\begin{array}{l}\text { Lycaena } \\
\text { dorcas }\end{array}$ & $\begin{array}{l}\text { Boloria } \\
\text { montinus }\end{array}$ \\
\hline Alsike clover Trifolium hybridum & $\mathrm{X}$ & & $\mathrm{X}$ \\
\hline Birdfoot trefoil Lotus corniculatus & $\mathrm{X}$ & $\mathrm{X}$ & $\mathrm{X}$ \\
\hline Black medick Medicago lupulina & $\mathrm{X}$ & $\mathrm{X}$ & \\
\hline Canada thistle Cirsium arvense & $\mathrm{X}$ & $\mathrm{X}$ & $\mathrm{X}$ \\
\hline Orange hawkweed Hieracium aurantiacum & & & $\mathrm{X}$ \\
\hline $\begin{array}{l}\text { Ox-eye daisy Chrysanthemum } \\
\text { leucanthemum }^{\mathrm{a}}\end{array}$ & $\mathrm{X}$ & $\mathrm{X}$ & $\mathrm{X}$ \\
\hline Rabbitfoot clover Trifolium arvense & $\mathrm{X}$ & $\mathrm{X}$ & $\mathrm{X}$ \\
\hline Red clover Trifolium pratense & & $X$ & $X$ \\
\hline Spotted knapweed Centaurea maculosa & $\mathrm{X}$ & & $X$ \\
\hline Yarrow Achillea millefolium & $X$ & $X$ & $X$ \\
\hline Yellow sweet clover Melilotus officinalis & $\mathrm{X}$ & & \\
\hline
\end{tabular}

${ }^{a}$ One B. eunomia informally observed in an upland roadside nectared on this

\section{Discussion}

\section{Characterization of bog butterfly fauna}

Nekola (1998) reported significantly different bog butterfly faunas in the three different bog vegetation types. Even with many more years of surveys, our results on which species occurred in which bog types are remarkably similar to Nekola's (1998) (Table 4). The minor differences in fauna between Nekola (1998) and us are easily attributable to species accumulation as a function of survey effort (Rosenzweig 1992); more species ought to be 
Table 9 Immigrant individuals observed (obs) in the earlier and later survey periods during 2002-2009 in bogs (not roadsides), for total individuals (including unidentified Vanessa) and by species, with expected (exp) individuals based on survey effort in spring $(160.2 \mathrm{~h}, 49.9 \%)$ and summer $(161.0 \mathrm{~h}, 50.1 \%)$

\begin{tabular}{|c|c|c|c|c|c|}
\hline & \multicolumn{2}{|c|}{ Before 16 June } & \multicolumn{2}{|c|}{ After 15 June } & \multirow[t]{2}{*}{$P$} \\
\hline & obs & $\exp$ & obs & $\exp$ & \\
\hline Total individuals & 409 & 240 & 73 & 242 & 0 \\
\hline Danaus plexippus & 293 & 171 & 49 & 171 & 0 \\
\hline Vanessa virginiensis & 36 & 19 & 2 & 19 & 0 \\
\hline Vanessa atalanta & 23 & 15 & 8 & 16 & 0.007 \\
\hline Junonia coenia & 24 & 15 & 6 & 15 & 0.0019 \\
\hline Vanessa cardui & 10 & 5 & 0 & 5 & 0.0044 \\
\hline Pontia protodice & 3 & 1 & 0 & 2 & 0.0662 \\
\hline Euptoieta claudia & 1 & 1 & 1 & 1 & 0.4795 \\
\hline
\end{tabular}

Per Nielsen (1999), it is unlikely but not directly known that any of the three Vanessa species overwinter in Michigan, the state immediately east of Wisconsin

Table $10 \mathrm{~N}$ individuals per year, by subgroups and total, observed (obs) in central and northern Wisconsin bogs (not roadsides) during 2002-2009, and expected (exp) individuals proportional to survey effort (h) per year. Each subgroup and total individuals deviated significantly from expected (Chi Square Goodness of Fit $P=0.0000)$

\begin{tabular}{|c|c|c|c|c|c|c|c|c|c|c|c|c|}
\hline \multirow[b]{2}{*}{ Year } & \multicolumn{2}{|c|}{ Survey effort } & \multicolumn{2}{|c|}{ Specialist } & \multicolumn{2}{|c|}{ Affiliate } & \multicolumn{2}{|c|}{ Generalist } & \multicolumn{2}{|c|}{ Immigrant } & \multicolumn{2}{|l|}{ Total } \\
\hline & $\mathrm{h}$ & $\%$ & Obs & Exp & Obs & Exp & Obs & Exp & Obs & Exp & Obs & Exp \\
\hline 2002 & 28.41 & 8.8 & 452 & 635 & 697 & 513 & 649 & 255 & 15 & 43 & 1974 & 1546 \\
\hline 2003 & 32.78 & 10.2 & 598 & 732 & 885 & 592 & 183 & 295 & 10 & 49 & 1861 & 1784 \\
\hline 2004 & 38.27 & 11.9 & 678 & 855 & 297 & 692 & 189 & 344 & 10 & 57 & 1242 & 2083 \\
\hline 2005 & 38.6 & 12 & 886 & 862 & 199 & 697 & 194 & 347 & 23 & 58 & 1369 & 2111 \\
\hline 2006 & 40.6 & 12.6 & 652 & 907 & 443 & 735 & 393 & 365 & 151 & 61 & 1711 & 2215 \\
\hline 2007 & 46.37 & 14.4 & 1061 & 1036 & 966 & 838 & 466 & 417 & 256 & 70 & 2935 & 2524 \\
\hline 2008 & 41.52 & 12.9 & 1241 & 928 & 1281 & 750 & 304 & 373 & 6 & 62 & 3095 & 2260 \\
\hline 2009 & 54.7 & 17 & 1609 & 1222 & 1037 & 988 & 510 & 492 & 11 & 82 & 3300 & 2978 \\
\hline
\end{tabular}

found with more visits in more years. European bog Lepidoptera communities exhibit very large differences between different bog structures, different parts of bogs, and between opener bogs and immediately adjacent wet forest (Spitzer et al. 1999; Dapkus 2004a, 2004b).

Nekola (1998) reported significantly fewer bog butterfly species in smaller bogs (muskegs and kettleholes only), but no difference in species richness among the three bog types when controlling for site size. We found that northern Wisconsin bogs were not depauperate in specialists compared to large barrens and heaths in the same region (cf. Table 5, 6). Furthermore, a number of bog specialists frequently occurred in numerous examples of bogs, including all three types (Table 7).

As reported for tyrphobiontic Lepidoptera elsewhere (Väisänen 1992; Spitzer et al. 1999; Dapkus 2004a), specialist species here comprised a small proportion (10\%) of all species recorded in bogs (Table 2), similar to the proportion of specialists in three tallgrass prairie subregions (9-16\%) and Wisconsin barrens (11\%) (Swengel 1998a). However, 
specialists and affiliates (tyrphophiles) are often the most abundant species in bogs (Väisänen 1992; Spitzer et al. 1999; Dapkus 2004a). In our study, four of the eight specialists were among the six most abundant butterfly species in bogs, out of 77 species recorded (Table 2). Six of the seven most abundant species were bog affiliate and specialist butterflies treated in Nekola (1998) as peatland-obligate species (cf. Table 4).

Specialists accounted for nearly half the total individuals observed in bogs (Table 3). By contrast, only $6 \%$ of individuals were specialists in the most fragmented tallgrass prairie subregion, and only $11 \%$ in the subregion with the largest patches, while the subregion with both relatively large patches and the most favorable management had 56\% specialist individuals (but the seasonal sampling period was the narrowest here, timed for peak specialist numbers) (Swengel and Swengel 2001). Wisconsin barrens (also less fragmented) had 46\% specialists (Swengel and Swengel 2001). High fragmentation in a relatively natural landscape due to long-term climatic variation (northern Wisconsin bogs) has more favorable outcomes for specialist butterfly abundance than anthropogenically highly fragmented vegetation (tallgrass prairie). This appears attributable to the high longterm stability of bog vegetation (when relatively undegraded by human activity) (see "Introduction") that is highly resistant to infiltration by vegetation in the surrounding landscape.

The use of non-native nectar in lowland roadsides by the summer specialists (Table 8) represents a very limited opportunism. The three summer species frequented adjacent lowland roadsides but virtually no individuals of any specialists occurred in adjacent uplands (Table 2). Thus, these species did not in any numbers follow this nectar availability into uplands, where these non-native (as well as native) nectar plants also occur widely.

Vegetative versus resource-based approach to defining habitat

The vegetative approach to defining habitats may be called the "ecosystem" approach but it's typically focused on floristic composition (Wisconsin Department of Natural Resources 1995, Packard and Mutel 1997; Panzer and Schwartz 1998). This approach has value: some bog specialist butterflies have remarkable frequency of occurrence in northern Wisconsin bogs (Table 9). This faunistic similarity of specialists across these bogs may be particularly pronounced due to the long-term stability typical of this vegetation and remarkably pristine condition of these sites (see "Introduction"). "Characteristic" butterflies are frequently identified for "zones" or "biomes" (e.g., Layberry et al. 1998, pp 9-11); on that large scale, these are typically "matrix" butterflies of a general vegetation type. But even in highly destroyed and fragmented tallgrass prairie, characteristic specialists (if in range) occurred in many examples of that vegetation (Speyeria idalia in Missouri and Minnesota, Oarisma poweshiek in Minnesota) (Swengel 1998b; Swengel and Swengel 1999a, 1999b). Thus, vegetative classifications are efficacious at grouping insects by their floristic associations (e.g., Panzer and Schwartz 1998; Shuey 2005).

Confounding the fit of butterflies to vegetative classifications is the single vegetative label typically assigned to a particular patch. Numerous non-specialist (non-tyrphobiontic) species associated with other types of vegetations occur in bogs (Table 2). In other words, a northern Wisconsin bog is also a heath that's wet (Colias interior), a peaty sedge meadow (Satyrodes eurydice), a particularly damp grassland (Coenonympha tullia) or meadow (Boloria selene), and a forest however scraggly (Erynnis icelus, Speyeria atlantis). Even many tyrphobiontic species occur there not because it's a bog (wetland) but because it's adequately analogous climatically and vegetatively to taiga or tundra (Spitzer and Danks 
2006). At the central Wisconsin pine barrens (Bauer-Brockway) richest in specialist butterfly species in our study (Swengel 1998a), we can record (in season) in one small location specialists of grassland (Hesperia metea, Atrytonopsis hianna, H. leonardus) and savanna (Callophrys irus, C. henrici) as well as forest-specific species (Megisto cymela, Enodia anthedon) (Swengel 2009). This is easily explained by the resource-based approach to defining habitat (Dennis and Eales 1997; Thomas et al. 2001; Dennis et al. 2007; Dennis 2010): each species finding the conditions and resources required. Conservation management decisions can foster or reduce this layering of vegetation types and associated insect diversity on top of each other (Kirby 1992). For example, tree-cutting can maintain a savanna (instead of grassland or forest) at the scale of a site but result in primarily grassland and forest subsites within that site (dissociating the grassland and forest butterflies, and leaving very little partially shaded vegetation for savanna butterflies) or maintain the mix throughout the site at the microsite scale. It is also more difficult to assert that species are co-evolved with a specific regime of processes particular or unique to each ecosystem (e.g., Wisconsin Department of Natural Resources 1995; Packard and Mutel 1997; Panzer 2002) when a site can have more than one ecosystem layered right on top of each other (Kirby 1992).

General ecosystems versus site individuality

Aiming for "ecosystems" in conservation management and restoration (Wisconsin Department of Natural Resources 1995; Packard and Mutel 1997; Panzer 2002) is aiming for a native but general vegetation type. This can lead to a more generalist fauna with the loss of specialists (e.g., Kirby 1992; Swengel 1996; Longcore et al. 2000; Nekola 2002). The primary method for prairie conservation management is burning, and this shift can be explained away (sites too small, too degraded) and blamed on the specific method of fire (fires too big, too frequent, and taking away from investing in other kinds of management). But in addition to those factors, an even more fundamental issue is aiming for the average and general ecosystem. Although native, this can lead to average and general butterflies. Bog butterflies reliably live only in sites persistently far outside the landscape average, even in a relatively natural northern Wisconsin context. An alternative approach to both site selection and management embraces site and species individuality by targeting specialists first. For example, by picking spots for the most specialized and rare birds first, then working up from there, all bird species were quickly captured in the fewest sites, compared to other methods of site selection (Williams et al. 1996). By logic, these sites should be conserved for their uniqueness, not be made more typical or average, even if also natural.

\section{Dynamism versus stability}

To be sure, bogs are particularly long-lived stable vegetation. Other vegetations are naturally more dynamic, so that conservationists aim to conserve and restore that dynamism. But pockets of remarkable stability are natural in other vegetations as well. Brown (1997) described paleo-environments in the tropics particularly speciose in the most conservative insect species, where surprisingly small perturbations of pristine vegetation might have permanent negative effects on those species. In a study of Canadian boreal forest (Gandhi et al. 2001), fire skips ("residuals") within the perimeter of the most recent wildfire contained older trees than in the unburned forest surrounding the most recent wildfire. The trees in the skips were on average 180 years old (maximum over 300 years old), while the surrounding mature forest unburned in the last fire was only about 72 years old. These fire 
skips were reservoirs for forest beetles, and the only place where a glacial relict beetle was found. These stably consistent pockets occur in other vegetation types, which also need to be conserved for insects there.

Gandhi et al. (2003) compared the beetle fauna in the fire skips to "residuals" (or "skips") within a recent clearcut. The clearcut residuals weren't selected for being "oldgrowth" and unsurprisingly, the clearcut skips didn't have the fauna of the wildfire skips. These results do however suggest that clearcut skips could be made more effective for conservation by targeting old-growth (not merely mature) forest. Insects have been proposed as indicators of many things (as reviewed in McGeoch 2007), but a particularly useful property of species groups with adequate knowledge of their ecology would be indication of these outlier paleo-environments not otherwise as easily discerned by plant composition and structure alone. A corollary to Haldane's possibly apocryphal quip about the creator's "inordinate fondness for beetles" (as repeated in Ashworth 2001) is an inordinate fondness for specialists (and thus the stability most likely to favor persistence of such faunas), at least given proclivities for landscape dynamism both in the non-conserved modern landscape and in ecological conservation management.

More continuous and unintensive managements (e.g., light grazing) and consistent managements, even if somewhat more intensive (e.g., biennial haying), are more favorable for specialist insects than either intensive or inconsistent managements (Kirby 1992). In rural Sweden, historical land use over the last two centuries was more effective than current land use at explaining which plant species currently lived in the grasslands (Gustavsson et al. 2007). While long-term grazing produced the most favorable floristic results currently, a consistent use of haying throughout the entire period was more favorable than switching from haying to grazing, even decades ago. Thus, conservation management needs to be retrospective to before preservation in embracing site stability (Whitehouse 2006), rather than only forward-looking after preservation and restoration begin. Attempting to turn the clock back to before anthropogenic degradation (or before a switch to less favorable management such as haying in Sweden) can do more harm than embracing and managing to maintain the semi-natural condition of the site now (Kirby 1992). Relatively more stable site histories (e.g., long-term occupancy and cutting by beaver Castor canadensis) also occur for patches occupied by species such as Gillett's checkerspot (Euphydryas gilletti) well known to inhabit patches generated in a dramatic cyclical way (stand-replacing fire) (Williams 1988). In conserved semi-natural vegetations, more consistent management (grazing) may produce higher relative numbers of localized insects than more dramatic, rotational management (Kirby 1992; Thomas and Harrison 1992).

Plants versus landscape consistency causing insects

It is axiomatic that increased plant diversity, especially native, increases insect biodiversity, from gardens to nature reserves (e.g., Panzer and Schwartz 1998; Burghardt et al. 2009). The mechanism for this is not, however, simply plants causing insects, a concept which results in a focus on floristic management and restoration (ecosystems) as the best way to conserve the insect species most associated with those plants. Instead, appropriate, consistent long-term landscape conditions cause both the plants and the associated insects. Just as plants and insects got "sunk and dunked" together in temperate-zone bogs as relicts due to climatic oscillations (Dapkus 2004a; Spitzer and Danks 2006; Whitehouse et al. 2008), so too only insects finding consistent resources in the surrounding landscape exist to benefit when native plants are restored to a garden or reserve. Whatever shortfalls of such 
resource consistency determine what insects do not benefit from such plantings. A focus on plants can lead to restoration that destroys the continuity of required resources in the process, and loses the associated insects (Kirby 1992), usually the ones most restricted to that site in the first place (such as described in Whitehouse et al. 2008). An alternate approach focuses on what's "right" about those plants and conditions now (what's been adequately consistent, however minimally, in resources to maintain such insect faunas), and maintaining that consistency, even if there are "wrong" things too.

Acknowledgments We greatly appreciate Mrs. Sandra McKibben and Drs. William and Elsa Boyce for funding our bog surveys. We also thank them, Jed Bromfield and Henya Rachmiel, U.S. Fish and Wildlife Service, and Wisconsin Department of Natural Resources for funding some barrens surveys. We thank Jeff Nekola for generously sharing tips, site locations, patch sizes, and help with plant identification. We greatly appreciate helpful comments from the referees and Editor-in-Chief David Hawksworth.

Open Access This article is distributed under the terms of the Creative Commons Attribution Noncommercial License which permits any noncommercial use, distribution, and reproduction in any medium, provided the original author(s) and source are credited.

\section{References}

Ashworth AC (2001) Chapter 8: perspectives on quaternary beetles and climate change. In: Gerhard LC, Harrison WE, Hanson BM (eds) Geological perspectives of global climate change. American Association of Petroleum Geologists Studies in Geology \#47, Tulsa, pp 153-168

Brown KS (1997) Diversity, disturbance, and sustainable use of Neotropical forests: insects as indicators for conservation monitoring. J Insect Conserv 1:25-42

Burghardt KT, Tallamy DW, Shriver WG (2009) Impact of native plants on bird and butterfly diversity in suburban landscapes. Conserv Biol 23:219-224

Cassie B, Glassberg J, Swengel A, Tudor G (2001) North American Butterfly Association (NABA) checklist and English names of North American butterflies, 2nd edn. North American Butterfly Association, Morristown

Curtis JT (1959) The vegetation of Wisconsin: an ordination of plant communities. Univ of Wisconsin Press, Madison

Dapkus D (2004a) Lepidoptera of a raised bog and adjacent forest in Lithuania. Eur J Entomol 101:63-67

Dapkus D (2004b) Macrolepidoptera in Laukenai and Notigalè raised bogs (Lithuania). Latv Entomol 41:52-59

Dennis RLH (2010) A resource-based habitat view for conservation: butterflies in the British landscape. Wiley-Blackwell, Oxford

Dennis RLH, Eales HT (1997) Patch occupancy in Coenonympha tullia (Müller, 1764) (Lepidoptera: Satyrinae): habitat quality matters as much as patch size and isolation. J Insect Conserv 1:167-176

Dennis RLH, Shreeve TG, Sheppard DA (2007) Species conservation and landscape management: a habitat perspective. In: Stewart AJA, New TR, Lewis OT (eds) Insect conservation biology: proceedings of the Royal Entomological Society's 23rd Symposium. CABI, Oxfordshire, Cambridge, pp 92-126

Ferge L (1992) 1991 Wisconsin Lepidoptera season summary. Newsl Wisc Entomol Soc 19(1):5-7

Gandhi KJK, Spence JR, Langor DW, Morgantini LE (2001) Fire residuals as habitat reserves for epigaeic beetles (Coleoptera: Carabidae and Staphylinidae). Biol Conserv 102:131-141

Gandhi KJK, Spence JR, Langor DW et al (2003) Harvest retention patches are insufficient as stand analogues of fire residuals for litter-dwelling beetles in northern coniferous forests. Can J For Res 34:1319-1331

Glassberg J (1999) Butterflies through binoculars: the East. Oxford Univ Press, New York

Gustavsson E, Lennartsson T, Emanuelsson M (2007) Land use more than 200 years ago explains current grassland plant diversity in a Swedish agricultural landscape. Biol Conserv 138:47-59

Hoffman RM (2002) Wisconsin's natural communities: how to recognize them, where to find them. Univ of Wisconsin Press, Madison

Kirby P (1992) Habitat management for invertebrates: a practical handbook. Royal Society for the Protection of Birds, Sandy

Kuehn RM (1983) New Wisconsin butterfly records. J Lepid Soc 37:228-235 
Layberry RA, Hall PW, LaFontaine JD (1998) The butterflies of Canada. Univ of Toronto Press, Toronto, Buffalo, and London

Longcore T, Mattoni R, Pratt G, Rich C (2000). On the perils of ecological restoration: lessons from the El Segundo blue butterfly. In: Keeley JE, Baer-Keeley M, Fotheringham CJ (eds) 2nd Interface between ecology and land management. US Geol Surv Open-File Report 00-62, Sacramento, pp 281-286

McGeoch M (2007) Insects and bioindication: theory and practice. In: Stewart AJA, New TR, Lewis OT (eds) Insect conservation biology: proceedings of the Royal Entomological Society's 23rd Symposium. CABI, Oxfordshire, Cambridge, pp 144-174

Nekola JC (1998) Butterfly (Lepidoptera: Lycaenidae, Nymphalidae, and Satyridae) faunas of three peatland habitat types in the Lake Superior drainage basin of Wisconsin. Great Lakes Entomol 31:27-38

Nekola JC (2002) Effects of fire management on the richness and abundance of central North American grassland snail faunas. Anim Biodiv Conserv 25(2):53-66

Nekola JC, Kraft CE (2002) Spatial constraint of peatland butterfly occurrences within a heterogeneous landscape. Oecologia 130:53-61

Nielsen M (1999) Michigan butterflies and skippers. Michigan State Univ Extension, East Lansing

Opler PA (1992) A field guide to eastern butterflies. Houghton Mifflin, New York

Opler PA, Krizek GO (1984) Butterflies east of the Great Plains. Johns Hopkins Univ Press, Baltimore and London

Packard S, Mutel CF (1997) The tallgrass restoration handbook: for prairies, savannas, and woodlands. Island Press, Washington and Covelo

Panzer R (2002) Compatibility of prescribed burning with the conservation of insects in small, isolated prairie reserves. Conserv Biol 16:1296-1307

Panzer R, Schwartz MW (1998) Effectiveness of a vegetation-based approach to insect conservation. Conserv Biol 12:693-702. doi:10.1046/j.1523-1739.1998.97051.x

Pollard E (1977) A method for assessing changes in abundance of butterflies. Biol Conserv 12:115-133

Riegler M (1995) Development of a pine barrens recovery plan. In: Borgerding, EA, Bartelt GA, McCown MA (eds) The future of pine barrens in northwest Wisconsin: a workshop summary. Wisconsin Dept Nat Res PUBL-RS-913-94, pp 28-33

Rosenzweig ML (1992) Species diversity gradients: we know more and less than we thought. J Mammal 73:715-730

Samson F, Knopf F (1994) Prairie conservation in North America. Bioscience 44:418-421

Schtickzelle N, Mennechez G, Baguette M (2006) Dispersal depression with habitat fragmentation in the bog fritillary butterfly. Ecology 87:1057-1065

Shuey JA (2005) Assessing the conservation value of a complementary system of habitat reserves relative to butterfly species at risk and divergent populations. Am Midl Nat 153:110-120

Spencer S, Collins S (2008) Reversing the decline in butterflies and moths across Europe-the importance of particular farming practices and the implications for CAP reform. www.birdlife.eu/eu/pdfs/BC Europe_CAPreformpaperFeb08.pdf. Viewed 15 Jan 2010

Spitzer K, Danks HV (2006) Insect biodiversity of boreal peat bogs. Annu Rev Entomol 51:137-161

Spitzer K, Bezdĕk A, Jaroš J (1999) Ecological succession of a relict Central European peat bog and variability of its insect biodiversity. J Insect Conserv 3:97-106

Swengel AB (1996) Effects of fire and hay management on abundance of prairie butterflies. Biol Conserv $76: 73-85$

Swengel AB (1998a) Comparison of butterfly richness and abundance measures in prairie and barrens. Biodiv Conserv 7:1639-1659

Swengel AB (1998b) Effects of management on butterfly abundance in tallgrass prairie and pine barrens. Biol Conserv 83:77-89

Swengel A (2009) The beguiling butterflies of the Jackson County pine-oak barrens. Southern Wisconsin Butterfly Association, Madison, Wisconsin. http://www.naba.org/chapters/nabawba/watching.html. Viewed 11 Feb 2010

Swengel AB, Swengel SR (1997) Co-occurrence of prairie and barrens butterflies: applications to ecosystem conservation. J Insect Conserv 1:131-144

Swengel A, Swengel S (1999a) Observations on prairie skippers (Oarisma poweshiek, Hesperia dacotae, H. Ottoe, H. leonardus pawnee, and Atrytone arogos iowa) (Lepidoptera: Hesperiidae) in Iowa, Minnesota, and North Dakota during 1988-1997. Great Lakes Entomol 32:267-292

Swengel SR, Swengel AB (1999b) Correlations in abundance of grassland songbirds and prairie butterflies. Biol Conserv 90:1-11

Swengel AB, Swengel SR (2001) Effects of prairie and barrens management on butterfly faunal composition. Biodiv Conserv 10:1757-1785 
Swengel AB, Swengel SR (2005) Long-term population monitoring of the Karner Blue (Lepidoptera: Lycaenidae) in Wisconsin, 1990-2004. Great Lakes Entomol 38:107-134

Swengel AB, Swengel SR (2007) Benefit of permanent non-fire refugia for Lepidoptera conservation in fire managed sites. J Insect Conserv 11:263-279

Swengel AB, Swengel SR (2010) High and dry or sunk and dunked: lessons for tallgrass prairies from quaking bogs. J Insect Conserv. doi:10.1007/s10841-010-9335-x

Thomas CD, Harrison S (1992) Spatial dynamics of a patchily distributed butterfly species. J Anim Ecol 61:437-446

Thomas JA, Bourn NAD, Clarke RT et al (2001) The quality and isolation of habitat patches both determine where butterflies persist in fragmented landscapes. Proc R Soc Lond B 268:1791-1796

Turlure C, Van Dyck H, Schtickzelle N, Baguette M (2009) Resource-based habitat definition, niche overlap and conservation of two sympatric glacial relict butterflies. Oikos 118:950-960

Väisänen R (1992) Distribution and abundance of diurnal Lepidoptera on a raised bog in southern Finland. Ann Zool Fennici 29:75-92

van Swaay CAM, Warren MS, Loïs G (2006) Biotope use and trends of European butterflies. J Insect Conserv 10:189-209

Vandewoestijne S, Baguette M (2004) Genetic population structure of the vulnerable bog fritillary butterfly. Hereditas 141:199-206

Whitehouse NJ (2006) What can forest managers learn from research on fossil insects? Linking forest ecological history, biodiversity and management. In: Grove SJ, Janula JL (eds) Insect biodiversity and dead wood: proceedings of a symposium for the 22nd International Congress of Entomology. Gen Tech Rep SRS-93. USDA Forest Service, Southern Research Station, Asheville, pp 30-41

Whitehouse NJ, Langdon PG, Bustin R, Galsworthy S (2008) Fossil insects and ecosystem dynamics in wetlands: implications for biodiversity and conservation. Biodiv Conserv 17:2055-2078

Williams EH (1988) Habitat and range of Euphydryas gillettii (Nymphalidae). J Lepid Soc 42:37-45

Williams P, Gibbons D, Margules C et al (1996) A comparison of richness hotspots, rarity hotspots, and complementary areas for conserving diversity of British birds. Conserv Biol 10:155-174

Wisconsin Department of Natural Resources (1995) Wisconsin's biodiversity as a management issue. Dept of Nat Res, Madison 